\title{
The Effectiveness of a New Topical Formulation Containing GSH-C4 and Hyaluronic Acid in Seborrheic Dermatitis: Preliminary Results of an Exploratory Pilot Study
}

This article was published in the following Dove Press journal:

Clinical, Cosmetic and Investigational Dermatology

\section{Elena Campione (D) \\ Sara Mazzilli \\ Caterina Lanna (D) \\ Terenzio Cosio \\ Vincenzo Palumbo \\ Gaia Cesaroni (iD \\ Flavia Lozzi $\mathbb{D}$ \\ Laura Diluvio \\ Luca Bianchi}

Dermatologic Unit, Department of Systems Medicine, University of Rome Tor Vergata, Rome, Italy
Correspondence: Elena Campione Dermatologic Unit, Department of Systems Medicine, University of Rome Tor Vergata, Viale Oxford 8I, Rome 00133 , Italy

Tel +390620902743

Email campioneelena@hotmail.com
Introduction: Seborrheic dermatitis is a common skin disease with clinical aspects similar to those of psoriasis, eczema or allergic reactions, appearing on the sebum-rich areas of the scalp, face, and trunk. Yeast like Malassezia species, immunologic abnormalities and activation of complement are recognized as a crucial pathogen for the onset of seborrheic dermatitis. Intermittent and active phases are characterized by burning, scaling and itching, then followed by inactive periods. The disease is sometimes severe up to the erythrodermia; thus, it has a great influence on the patient's quality of life. In vitro and vivo studies have shown that the exogenous intake of glutathione-GSH-C4 and tocopherol inhibits lipid peroxidation and effectively fights and reduces oxidative stress in inflammatory disorders.

Methods: We have carried out a study enrolling 20 patients affected by SD to evaluate the effectiveness and tolerability of a new topical formulation in cream (hereinafter SEB) containing GSH-C4 $0.4 \%$ in hyaluronic acid $0.25 \%$ - a new synthetic glutathione derivate called INCI (butyroyl glutathione)-assigned by the Personal Care Council. Investigator Global Assessment score and Patient Global Assessment of Treatment scales were used to test the efficacy of this new formulation.

Results: All patients showed a good clinical response to the treatment with topical SEB demonstrated by the gradual reduction in inflammatory skin lesions.

Discussion: The results of our pilot study confirm the efficacy and tolerability of this new topical formulation in a real-life assessment and patients showed strong adherence to therapy. These promising results - still to be confirmed on a larger number of patients - emphasize the potential SEB has in controlling the chronic inflammation of seborrheic dermatitis.

Keywords: seborrheic dermatitis, glutathione, pilot study

\section{Introduction}

Seborrheic dermatitis (SD) is an inflammatory disease present in $1-3 \%$ of the population, more frequent in men than in women, mostly occurring in adolescents and young adults. Clinical manifestations consist in erythematous, yellowish and desquamating patches and plaques in the scalp, eyebrows, ears, nasolabial folds, chest, axillae and inguinal area. ${ }^{1}$ Diagnosis is predominantly based on clinical signs.

Its etiology is still unknown; nevertheless, SD needs endogenous and exogenous predisposing factors for its development. ${ }^{2,3}$ 
The hormonal imbalance, mainly of androgens, seems an important predisposing factor, as suggested by the characteristic localization of SD lesions (sebaceous glandrich body areas target for androgens), age (adolescence) and sex (2:1 for men) prevalence of the manifestations. ${ }^{2}$

Alterations in microbiota composition is also involved in the pathogenic process of SD. Microorganisms colonizing SD skin include yeasts, in particular - Malassezia globosa and restricta - and bacteria - Cutibacterium acnes, Micrococcus butyricus and Micrococcus pyogenes var. aureus. ${ }^{1,4}$

Moreover, SD is more common in immunosuppressed patients - especially if HIV positive - suggesting that immune mechanisms are involved in its development. Humoral and cellular immunity have been studied in patients with SD, though with conflicting results. ${ }^{5,6}$ Nonetheless, an alteration of immune responses has been described: NKs increase, low IgG titers, IL-2 and INF $\gamma$ reduction. ${ }^{6}$

Plasmatic polyunsaturated fatty acid (PUFA) deficits, sebaceous squalene and waxes reduction have often been found in patients with the copresence of HIV and SD. The plasma deficit of PL-PUFA is closely associated with reduced blood levels of vitamin $\mathrm{E}$, glutathione peroxidase and ubiquinone, ${ }^{7}$ suggesting a possible role of oxidative stress in SD development.

Indeed, mitochondrial functional impairment causing increased lipid peroxidation and oxidative state is significantly increased in patients with Parkinson's disease, Down's syndrome and cancer, all diseases in which SD is more frequently observed. ${ }^{8}$

Therefore, the involvement of free radicals and reactive oxygen species (ROS) could be an important key risk factor in the development of SD - as suggested and demonstrated by Emre et $\mathrm{al}^{8}$ - and a possible SD target-therapy.

Glutathione (GSH) is a non-protein molecule composed of three amino acids, cysteine, glutamic acid and glycine bound together. From a chemical point of view, it is a tripeptide, in which cysteine and glycine are bound together by a normal $\alpha$-peptide bond, while glutamate is bound to cysteine through a $\gamma$-peptide bond between the carboxylic group of the lateral chain of glutamate and the amino group of cysteine. In this way, the sulfhydryl group of cysteine can undergo oxidation and form a dimer with another molecule of oxidized glutathione - identified by the acronym GSSG binding through a sulfide bridge. The main protective characteristics of GSH as a defense mechanism against oxidative stress are related to the following properties: ${ }^{9}$ it is a cofactor of several detoxification enzymes against oxidative stress, such as glutathione peroxidase and glutathione transferase; it participates to the transport of amino acids through plasma membranes; it acts as a "scavenger", which literally sweeps hydroxides and singlet oxygen to detoxify hydrogen peroxide and lipoperoxides through the catalytic action of glutathione peroxidase. ${ }^{10}$

GSH is able to regenerate the most important antioxidants, vitamins $\mathrm{C}$ and $\mathrm{E}$ from their active forms and can reduce the radical tocopherol of vitamin $\mathrm{E}$ directly, or indirectly, through the reduction of semidehydroascorbate into ascorbate. ${ }^{11}$

Therefore, GSH, protecting cells against exogenous and endogenous harmful molecules including reactive oxygen and nitrogen species (ROS/RNS), ${ }^{3,4}$ could be used within this context as a therapeutic strategy against SD.

Limongi et al recently showed that a GSH derivate (N-butanoyl glutathione, GSH-C4) is able to penetrate cell membranes and buffer GSH depletion, thus counteract on the pro-inflammatory response in different cellular models of inflammation, by reducing the expression of pro-inflammatory cytokines via NFkB modulation. ${ }^{12}$

Taking into account this role of GSH-C4 and the importance of the redox state in the development of SD, we performed an exploratory open pilot study to investigate whether the topical use of GSH-C4 in hyaluronic acid, can influence SD evolution.

\section{Patients and Methods}

We performed an exploratory open pilot study involving 20 patients (13 men and 7 women) with SD on the face to evaluate the effectiveness of a topical formulation in cream containing GSH-C4 $0.4 \%$ in Hyaluronic acid $0.25 \%$ (hereinafter SEB).

Patients applied the cream twice a day for 15 days and then once a day for another 15 days. Patients with other dermatological disorders and patients treated with other topical therapies for SD in the previous month were excluded from the study.

Patients were evaluated at baseline and at the second and fourth weeks of treatment by means of a clinical severity score, which was calculated by dividing the face into three segments: forehead and eyebrows, cheeks and nose, chin. Clinical severity score was the sum of erythema and flaking determined for each part, between 0 and 3 ( 0 : absent, 1: mild, 2: moderate, 3 : severe).

In order to assess disease severity at baseline and after treatment, we applied two scales: Investigator Global Assessment (IGA) score by retrospectively reviewing clinical charts and photographs and Patient Global Assessment of 
Table I Clinical Evaluation Parameters

\begin{tabular}{|l|l|l|l|l|l|}
\hline Scale & 0-Absent & I-Mild & 2-Moderate & 3-Severe & 4-Very severe \\
\hline Erythema & 0-Absent & I-Mild & 2-Moderate & 3-Severe & 4-Very severe \\
\hline Pruritus & 0-Absent & I-Mild & 2-Moderate & 3-Severe & 4-Very severe \\
\hline Provider Global Assessment & 0-Clear & I-Almost clear & 2-Mild & 3-Moderate & 4-Severe \\
\hline
\end{tabular}

Treatment (PGA) based on 5-point Likert scale. For IGA, we rated overall severity on a 0 -to-4 scale as $01 / 4$ clear, $11 / 4$ almost clear, 2 1/4 mild, 3 1/4 moderate, and $41 / 4$ severe. We considered treatment to be successful when lesions cleared or were almost cleared (Table 1).

In addition, a Pruritus Visual Analogue Scale (PVAS) with a 10-point scale was checked for itching assessment, whilst a DLQI questionnaire with a 30-point scale was used to evaluate changes in the quality of life.

Both patients and physicians made a global evaluation at week 4 to evaluate the improvement achieved at the end of therapy as significant, moderate, mild or as absent.

Side effects such as pruritus, burning perception and erythema were all checked at each control visit.

The primary endpoint of the study was to evaluate effectiveness by measuring the achievement of clinical improvement in percentage.

The secondary endpoint was the evaluation of the tolerability of the SEB formulation and appreciation by the patients via DLQI assessment. ${ }^{13}$

After receiving an extensive disclosure of the study purposes, all patients gave written informed consent to have the case details and any accompanying images published. The study was performed according to the principles of Helsinki Declaration and approved by Policlinico Tor Vergata University Hospital Ethic Committee (N. Protocol: 116/17).

\section{Results}

All patients enrolled in this study completed the treatment; 10 patients were affected by severe SD, 7 by moderate and 3 by mild disease.

Subjects were considered successfully treated if the erythema and scaling as well as investigator global assessment (IGA) scores decreased to 0 (or 1 if the baseline score was $\geq 3$ ).

All patients showed a good clinical response to SEB treatment with a gradual decline in inflammatory skin lesions. Complete or almost complete clearance (IGA score $0-1$ ) was achieved in 20 cases. Ten patients with severe DS achieved IGA $0-1$ after 4 weeks of treatment, 7 moderate patients after 4 weeks, 3 patients after 2 weeks (Figures 1 and 2).
Patients achieved complete recovery from SD, presenting a marked improvement in symptoms even after the first week of therapy with a decrease in pruritus confirmed via VAS score. Eighteenpatients achieved complete improvement of itching symptoms (VAS 0), whilst 2 patients almost completely solved their pruritus after 2 weeks of topical application of the SEB formulation (Table 2)

Patients reported complete reduction in DLQI score after 2 weeks of treatment and showed no SD recurrence after 2 months from the end of treatment.

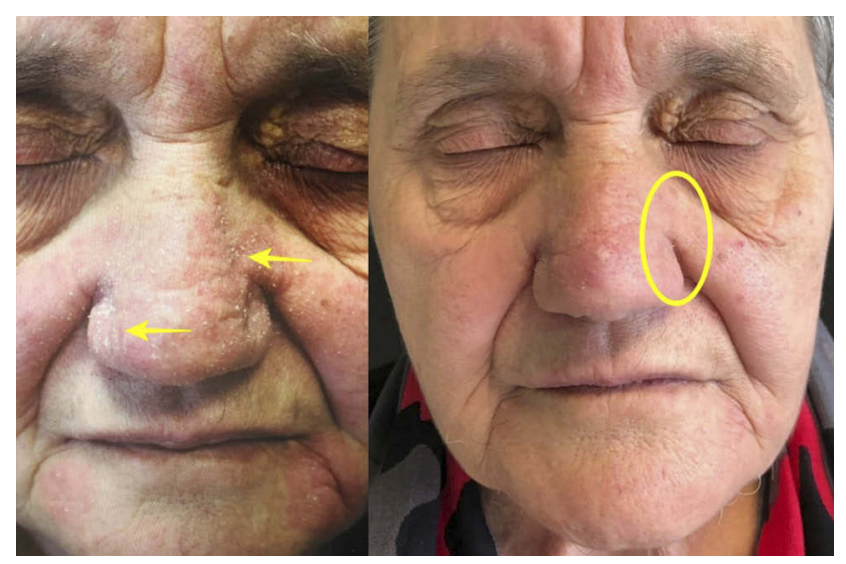

Figure I Patient at baseline T0 and T4. In the yellow circle it is possible to see the complete regression of the inflamed and desquamated area (yellow arrows).

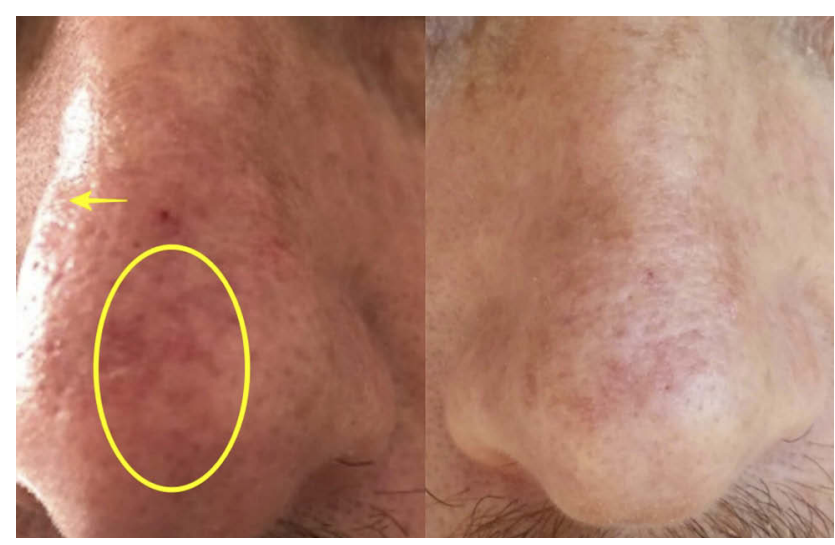

Figure 2 Patient at T0 (left) and T4 (right). Here we can notice the local inflammation has reduced (yellow circle), whilst the skin watermark has improved (yellow arrows). 
Table 2 Investigator Global Assessment (IGA) Score by Retrospective Review of Clinical Charts and Photographs and Patient Global Assessment of Treatment (PGA) Based on 5-Point Likert Scale

\begin{tabular}{|c|c|c|c|c|c|c|c|c|c|c|}
\hline & & $\begin{array}{l}\text { Medium } \\
\text { IGA Score } \\
\text { T0 } \\
\text { (Median) }\end{array}$ & $\begin{array}{l}\text { Medium } \\
\text { IGA Score } \\
\text { T2 } \\
\text { (Median) }\end{array}$ & $\begin{array}{l}\text { Medium } \\
\text { IGA Score } \\
\text { T4 } \\
\text { (Median) }\end{array}$ & $\begin{array}{l}\text { Medium } \\
\text { PVAS } \\
\text { Score T0 } \\
\text { (Median) }\end{array}$ & $\begin{array}{l}\text { Medium } \\
\text { PVAS } \\
\text { Score T2 } \\
\text { (Median) }\end{array}$ & $\begin{array}{l}\text { Medium } \\
\text { PVAS } \\
\text { Score T4 } \\
\text { (Median) }\end{array}$ & $\begin{array}{l}\text { Medium } \\
\text { DLQI } \\
\text { Score T0 } \\
\text { (Median) }\end{array}$ & $\begin{array}{l}\text { Medium } \\
\text { DLQI } \\
\text { Score T2 } \\
\text { (Median) }\end{array}$ & $\begin{array}{l}\text { Medium } \\
\text { DLQI } \\
\text { Score T4 } \\
\text { (Median) }\end{array}$ \\
\hline $\begin{array}{l}\text { Total } \\
\text { patients }\end{array}$ & 20 & $\begin{array}{l}3.35 \\
(4)\end{array}$ & $\begin{array}{l}1.35 \\
(2)\end{array}$ & $\begin{array}{l}0,4 \\
(0)\end{array}$ & $\begin{array}{l}4.25 \\
(4)\end{array}$ & $\begin{array}{l}2.25 \\
(2)\end{array}$ & $\begin{array}{l}0,6 \\
(0)\end{array}$ & $\begin{array}{l}19.25 \\
(19)\end{array}$ & $\begin{array}{l}0 \\
(0)\end{array}$ & $\begin{array}{l}0 \\
(0)\end{array}$ \\
\hline $\begin{array}{l}\text { Male } \\
\text { (number) }\end{array}$ & $\begin{array}{l}65 \% \\
(13)\end{array}$ & $\begin{array}{l}2.46 \\
(3)\end{array}$ & $\begin{array}{l}0.65 \\
(2)\end{array}$ & $\begin{array}{l}0,2 \\
(0)\end{array}$ & $\begin{array}{l}4.08 \\
(4)\end{array}$ & $\begin{array}{l}2 \\
(2)\end{array}$ & $\begin{array}{l}0.35 \\
(0)\end{array}$ & $\begin{array}{l}20 \\
(20)\end{array}$ & $\begin{array}{l}0 \\
(0)\end{array}$ & $\begin{array}{l}0 \\
(0)\end{array}$ \\
\hline $\begin{array}{l}\text { Female } \\
\text { (number) }\end{array}$ & $\begin{array}{l}35 \% \\
(7)\end{array}$ & $\begin{array}{l}3.28 \\
(4)\end{array}$ & $\begin{array}{l}1.57 \\
(2)\end{array}$ & $\begin{array}{l}0.57 \\
(I)\end{array}$ & $\begin{array}{l}4.57 \\
(4)\end{array}$ & $\begin{array}{l}2.43 \\
(3)\end{array}$ & $\begin{array}{l}0.25 \\
(1)\end{array}$ & $\begin{array}{l}17.86 \\
(17)\end{array}$ & $\begin{array}{l}0 \\
(0)\end{array}$ & $\begin{array}{l}0 \\
(0)\end{array}$ \\
\hline
\end{tabular}

For IGA, we rated overall severity on a 0-to-4 scale as 0 I/4 clear, I I/4 almost clear, 2 I/4 mild, 3 I/4 moderate, and 4 I/4 severe. We considered treatment to be successful when lesions cleared or were almost cleared. Subjects were considered successfully treated if the erythema and scaling as well as investigator global assessment (IGA) scores decreased to 0 (or I if the baseline score was $>$ or $=3$ ).

\section{Discussion}

$\mathrm{SD}$ is a chronic relapsing skin condition that worsens due to alterations of the cutaneous microflora and redox imbalance. ${ }^{3}$ The current topical therapies can fail, and steroids cannot always be used for a long time due to local adverse effects.

It is therefore necessary to use new molecules with a more rapid mechanism of action since the pathology is evident in all visible areas and this has a negative influence on the patient's quality of life.

In our clinical experience with the use of topical SEB, we underline the importance of a new molecule acting on the pathogenetic pathway of SD. To our knowledge, this is the first study that explores the use of glutathione in SD.

GSH-C4 is a derivative of GSH obtained by combining peptide with n-butyric acid, used in a topic formulation. We synthetized a series of glutathione (GSH) derivatives with aliphatic chains of different lengths, coupled by peptides bound to the alpha-NH2 group of Glu. When added to several cell lines, the C6 (n-hexanoyl), C8 (n-octanoyl) and $\mathrm{C} 12$ (n-dodecanoyl) derivatives were toxic while the $\mathrm{C} 2$ (nethanoyl) and C4 (n-butanoyl) derivatives were not. ${ }^{14}$

The antioxidant activity of the derived n-butyric glutathione has been measured through the ORAC (oxygen radical absorbance capacity) test - as indicated by Ninfali et al - and was similar to that of reduced GSH. ${ }^{15}$

A study of 54 patients diagnosed with SD compared with 54 healthy controls, assessed oxidative stress status and measured serum total antioxidant status (TAS), total oxidative status (TOS) and oxidative stress index (OSI). The mean TAS values were significantly lower in the patient group $(\mathrm{P}=0.024)$ and patients had significantly higher TOS and OSI values compared to those of the control group $(\mathrm{P}=0.05)$.
There was no correlation between SD severity and TAS, TOS, and OSI values. The authors concluded that oxidative stress due to overproduction of oxygen radicals - reactive oxygen species or inadequate antioxidants - might contribute to the pathogenesis of SD. ${ }^{8}$ Reactive oxygen species (ROS) cause lipid peroxidation in the cell membrane, DNA damage and the secretion of inflammatory cytokines, eliciting an immune and inflammatory response. Endogenous antioxidants, such as glutathione (GSH) are used by activated immune cells to control their rising concentrations of ROS. However, in SD, as mentioned above, there is an increased, deregulated, activation of these cells, which leads to an increased production of ROS, impossible to control only through the endogenous amount of antioxidants. Thus, the exogenous application of GSH-C4 - as confirmed by the preliminary results of our study - that restores intracellular GSH levels could be a way to counteract ROS hyperproduction and the consequent inflammatory responses. ${ }^{3}$

Indeed, SEB showed great results and complete recovery without side effects, proving to be a new valid topical treatment for SD compared to other available topical therapies currently used in clinical practice.

Other therapeutic strategies include calcineurin inhibitors such as pimecrolimus, phototherapy, lithium gluconate/succinate, salicylic acid, selenium sulfide, sodium sulfacetamide, benzoyl peroxide. ${ }^{16}$

Previous studies analyzed the therapeutic role of hyaluronic acid. Schlesinger and Powell studied Hyaluronic acid sodium salt gel $0.2 \%$ as a topical device effective in reducing skin inflammation. They noticed that the provider global assessment improved from baseline in $92.3 \%$ of subjects. ${ }^{17}$ As reported, low molecular weight hyaluronic 
acid (LMWHA) fragments are biologically active as demonstrated by their ability to trigger built-in immune defense mechanisms and promote cytokine production. The effectiveness of substances applied on the surface of the epidermis depends on their ability to penetrate the stratum corneum. ${ }^{18}$ Moreover, a moist environment provided by LMWHA's hydrophilic nature can modify cellular behavior. Thus, we decided to use topical glutathione in order to have a synergy for reducing redox imbalance.

In the light of all these aspects, non-steroidal antiinflammatory agents such as GSH-C4, can be considered as the best topical therapy to treat scalp and non-scalp SD.

Indeed, GSH-C4 increases the level of endogenous antioxidants and can be considered as a natural anti-inflammatory product without any of those side effects linked to proper steroids. GSH-C4 cream can be used in every patient. There can be comorbidities for patients suffering from diabetes, Cushing disease or - most important of all - HIV positive patients.

Our experience suggests that the use of $0.4 \%$ local glutathione is safer than other formulations that may have a cytotoxic skin effect. SEB in its current concentration showed greater safety compared to higher values of glutathione in terms of skin cytotoxicity, skin irritation and dryness. The product was well tolerated and easily applied on the entire face without any side effects.

The results of our pilot study confirm the great efficacy and tolerability of this new molecule in a real-life assessment with patients showing adherence to therapy. However. we hope this can be a starting point to think about GSH and promote more research on its potential future application in $\mathrm{SD}$ or in other inflammatory skin diseases.

\section{Disclosure}

The authors report no conflicts of interest in this work.

\section{References}

1. Naldi L, Rebora A. Clinical practice. Seborrheic dermatitis. $N$ Engl J Med. 2009;360:387-396. doi:10.1056/NEJMcp0806464

2. Sampaio ALSB, Mameri ACA, Vargas TJDS, et al. Seborrheic dermatitis. An Bras Dermatol. 2011;86:1061-71; quiz 1072-4. doi:10.1590/s0365-05962011000600002

Clinical, Cosmetic and Investigational Dermatology

Publish your work in this journal

Clinical, Cosmetic and Investigational Dermatology is an international, peer-reviewed, open access, online journal that focuses on the latest clinical and experimental research in all aspects of skin disease and cosmetic interventions. This journal is indexed on CAS.
3. Dessinioti C, Katsambas A. Seborrheic dermatitis: etiology, risk factors, and treatments: facts and controversies. Clin Dermatol. 2013;31:343-351. doi:10.1016/j.clindermatol.2013.01.001

4. Prete SD, Angeli A, Ghobril C, et al. Anion inhibition profile of the $\beta$-carbonic anhydrase from the opportunist pathogenic fungus malassezia restricta involved in dandruff and seborrheic dermatitis. Metabolites. 2019;9:147. doi:10.3390/metabo9070147

5. Wikramanayake TC, Borda LJ, Miteva M, et al. Seborrheic dermatitis-looking beyond Malassezia. Exp Dermatol. 2019;28: 991-1001. doi:10.1111/exd.14006

6. Forrestel AK, Kovarik CL, Mosam A, et al. Diffuse HIV-associated seborrheic dermatitis - a case series. Int J STD AIDS. 2016;27: 1342-1345. doi:10.1177/0956462416641816

7. Passi S, De Luca C, Picardo M, et al. Deficit ematico di acidi grassi polinsaturi di fosfolipidi, vitamina $\mathrm{E}$ e glutatione perossidasi come possibile fattore di rischio nell'insorgenza e nello sviluppo della sindrome da immunodeficienza acquisita. [Blood deficiency values of polyunsaturated fatty acids of phospholipids, vitamin E and glutathione peroxidase as possible risk factors in the onset and development of acquired immunodeficiency syndrome]. G Ital Dermatol Venereol. 1990;125:125-130. Italian.

8. Emre S, Metin A, Demirseren DD, et al. The association of oxidative stress and disease activity in seborrheic dermatitis. Arch Dermatol Res. 2012;304:683-687. doi:10.1007/s00403-012-1254-0

9. Filomeni G, Rotilio G, Ciriolo MR. Cell signalling and the glutathione redox system. Biochem Pharmacol. 2002;64:1057-1064. doi:10.1016/s0006-2952(02)01176-0

10. Forman HJ, Zhang H, Rinna A. Glutathione: overview of its protective roles, measurement, and biosynthesis. Mol Aspects Med. 2009;30:1-12. doi:10.1016/j.mam.2008.08.006

11. Leedle RA, Aust SD. The effect of glutathione on the vitamin E requirement for inhibition of liver microsomal lipid peroxidation. Lipids. 1990;25:241-245. doi:10.1007/bf02544382

12. Limongi D, Baldelli S, Checconi P, et al. GSH-C4 acts as antiinflammatory drug in different models of canonical and cell autonomous inflammation through $\mathrm{NF \kappa B}$ inhibition. Front Immunol. 2019;10:155. doi:10.3389/fimmu.2019.00155

13. Poór AK, Brodszky V, Péntek M, et al. Is the DLQI appropriate for medical decision-making in psoriasis patients? Arch Dermatol Res. 2018;310:47-55. doi:10.1007/s00403-017-1794-4

14. Palamara AT, Brandi G, Rossi L, et al. New synthetic glutathione derivatives with increased antiviral activities. Antivir Chem Chemother. 2004;15:83-91. doi:10.1177/095632020401500204

15. Ninfali P, Antonini E, Frati A, et al. C-Glycosyl Flavonoids from Beta vulgaris Cicla and Betalains from Beta vulgaris rubra: antioxidant, anticancer and antiinflammatory activities-A review. Phytother Res. 2017;31:871-884. doi:10.1002/ptr.5819

16. Clark GW, Pope SM, Jaboori KA. Diagnosis and treatment of seborrheic dermatitis. Am Fam Physician. 2015;91:185-190.

17. Schlesinger T, Powell CR. Efficacy and safety of a low molecular weight hyaluronic acid topical gel in the treatment of facial seborrheic dermatitis final report. J Clin Aesthet Dermatol. 2014;7:15-18.

18. Gariboldi S, Palazzo M, Zanobbio L, et al. Low molecular weight hyaluronic acid increases the self-defense of skin epithelium by induction of beta-defensin 2 via TLR2 and TLR4. J Immunol. 2008;181:2103-2110. doi:10.4049/jimmunol.181.3.2103
The manuscript management system is completely online and includes a very quick and fair peer-review system, which is all easy to use. Visit http://www.dovepress.com/testimonials.php to read real quotes from published authors. 